\title{
Satisfacción de clientes externos. Estudio de caso de una piscina cubierta
}

\section{Satisfaction of external customers. A case study of an indoor swimming pool}

\author{
Cristina Sánchez García, Carmen María González Carcelén, Guillermo Felipe López Sánchez* y Arturo Díaz Suárez
}

Facultad de Ciencias del Deporte, Universidad de Murcia, España. Campus de Excelencia Internacional Regional "Campus Mare Nostrum".

\begin{abstract}
Resumen: Actualmente, las actividades acuáticas están en auge al ser muy demandadas por la población y los usuarios deportivos. El objetivo de este trabajo fue llevar a cabo un estudio de caso, valorando la satisfacción de los usuarios de la piscina municipal de Puente Tocinos (Región de Murcia). Para ello, se contó con una muestra compuesta por 44 usuarios (28 mujeres y 16 hombres), con una media de edad de 27,73 años (DT 14,5) y un rango de edad de 14 a 64 años. Todos los participantes cumplimentaron el "Cuestionario de valoración de servicios deportivos (EPOD2)”. Los datos obtenidos se analizaron con el programa informático SPSS 23. Los resultados mostraron una alta satisfacción general, sin diferencias significativas según sexo y edad. Los valores más altos de satisfacción se dieron en las dimensiones: monitores, actividades ofertadas, relación entre el personal de la instalación, y calidad vinculada al precio. Los valores más bajos correspondieron a la limpieza y amplitud de los vestuarios de la instalación. Se recomienda trabajar en la ampliación de espacios y limpieza de estos.

Palabras clave: natación, calidad percibida, instalaciones acuáticas, gestión deportiva.
\end{abstract}

Abstract: Nowadays, aquatic activities are very important because they are very demanded by the population and sport users. The aim of this study was to carry out a case study, assessing the satisfaction of the users of the public swimming pool of Puente Tocinos (Region of Murcia). For this purpose, the sample was composed of 44 users ( 28 females and 16 males), with a mean age of 27.73 years (SD 14.5), ranging from 14 to 64 years. All participants completed the "Sports Services Assessment Questionnaire (EPOD2)". The data obtained were analyzed using SPSS 23 software. The results showed a high overall satisfaction, without significant differences according to sex and age. The highest values of satisfaction were given in the dimensions: monitors, activities offered, relationship between the staff of the facility, and quality-price ratio. The lowest values corresponded to the cleanliness and spaciousness of the dressing rooms of the facility. It is recommended to work in the expansion and cleaning of spaces.

Key words: swimming, perceived quality, aquatic facilities, sport management.

\section{Introducción}

En el ámbito de la gestión deportiva son especialmente relevantes las investigaciones acerca de la satisfacción de los usuarios con los servicios e instalaciones deportivas (López, 2016; Martínez-Moreno, 2010). La satisfacción de los usuarios de servicios e instalaciones deportivas es un elemento fundamental para conseguir que los niveles de actividad física y deporte no sigan bajando, ya que según los últimos estudios, el nivel de actividad física de la población está muy por debajo del recomendado por la Organización Mundial de la Salud (López Sánchez et al, 2016 a, b, 2017; Smith, Fisher, \& Hamer, 2015; Vespalec, Pavlík, Zvonař, \& Zeman, 2016; Williams et al., 2017).

Además, la actividad física se presenta como alternativa saludable al consumo de sustancias nocivas y al estilo de vida sedentario (Castro et al., 2015; Chacón et al., 2016; Espejo et al., 2017). Por ello, el aumento de los servicios deportivos acuáticos justifica el interés por el conocimiento del público usuario, sus características, intereses y necesidades (Rial, Alonso, Rial, Picón, \& Varela, 2009).

La evolución sociodemográfica de la población ha producido un cambio provocando como consecuencia una ruptura con el modelo deportivo-competitivo (Durán, 1995 citado en Rodríguez, y Barriopedro, 2003). En los años 60 y 70, los

Dirección para correspondencia [Correspodence address]: Guillermo Felipe López Sánchez. E-mail: gfls@um.es intereses para acudir a las instalaciones acuáticas eran el rendimiento y competición (Puig, y Heinemann, 1991 citado en Rodríguez, y Barriopedro, 2003). Durante los años 80 y 90 surgieron otros tipos de demanda como pueden ser la salud, recreación o la diversión (Shostack, 1987 citado en Rodríguez y Barriopedro, 2003).

En esta línea, se ha producido un aumento de la demanda de ejercicio físico acuático, la cual ha favorecido el aumento de la oferta de programas de actividades físicas acuáticas dirigidas a la población (García Mayor et al., 2016).

En cuanto a lo que a satisfacción y calidad percibida en instalaciones deportivas se refiere, un estudio realizado en los centros deportivos del Ayuntamiento de Cartagena sobre la satisfacción como índice de calidad, señala que antiguamente, el índice de satisfacción de los usuarios era superior al que hubo en el año 2015. Por otro lado, se observa que la mejor calificación de los servicios deportivos viene dada por mujeres, de una edad comprendida entre 25-44 años y con ocupación laboral (Armada, Martínez-Gallego, Segarra, y Díaz, 2016).

Por otra parte, en la Universidad de Barcelona se analizó la satisfacción en relación a la profundidad del vaso. Se tuvo en cuenta la edad de los usuarios, el nivel de nado y los intereses que les conducen a la práctica de actividades acuáticas. Se observó cómo las personas adultas y mayores tienen una mayor predilección por el uso de un vaso de poca profundidad. Esto también ocurre en aquellos con un nivel bajo de nado, que 
prefieren un vaso de poca profundidad debido a la inseguridad que les genera no hacer pie en la piscina. En cambio, a los usuarios con un nivel de nado superior les resulta indiferente el nivel de profundidad. Por último, los usuarios que acuden a la piscina por cuestiones de salud y/o mantenimiento de la forma, que son la gran mayoría, se muestran más satisfechos en un vaso poco profundo que en los demás tipos de vaso (Rodríguez, y Barriopedro, 2003).

Tal y como se observa, hay numerosos estudios sobre herramientas para medir la satisfacción en instalaciones deportivas, tanto de usuarios como de técnicos. Cabe resaltar estudios recientes sobre la satisfacción de usuarios de gimnasios (Cuadrado et al., 2015; Rodríguez et al., 2015) y de clubes de pádel (Aparicio et al, 2016) también llevados a cabo en la Región de Murcia, así como de usuarios de servicios deportivos realizados en Alicante (García Mayor et al., 2016). Otros autores como Reynoso et al. (2015) llevaron a cabo un estudio sobre la satisfacción laboral de técnicos deportivos en Alicante. No obstante, no son tan numerosas las investigaciones específicas sobre la satisfacción de los usuarios de piscinas.

El objetivo del estudio es determinar la satisfacción de usuarios de piscina, a fin de diagnosticar las debilidades más comunes, para minimizar las deficiencias entre las expectativas del usuario y lo ofertado por parte de las empresas.

\section{Método}

Sujetos

La muestra en esta investigación estuvo constituida por los usuarios de natación de la Piscina Municipal de la localidad murciana de Puente Tocinos (Región de Murcia), durante el curso escolar 2016-2017. La muestra estuvo compuesta por 44 sujetos (28 de sexo femenino, 63.6\%; 16 de sexo masculino, $36.4 \%$ ), con una media de edad de 27,73 ańos (DT 14,5 ) y un rango de edad de 14 a 64 ańos.

\section{Procedimiento e Instrumento}

El procedimiento seguido ha comenzado con un análisis de la literatura científica al respecto. La técnica empleada ha sido la encuesta, utilizando como instrumento el "Cuestionario de valoración de servicios deportivos EPOD2" (Nuviala, et al., 2013).

El cuestionario consta de 25 ítems y de las siguientes 8 dimensiones: monitor/entrenador, instalaciones, material deportivo, actividades, comunicación, personal de la organización, satisfacción general, satisfecho con la relación calidad/ precio. Respecto a la fiabilidad, este cuestionario presenta una fiabilidad de 0.898 .

El mencionado cuestionario fue distribuido entre los suje- tos de la muestra, tras explicarles los objetivos de la investigación y las pertinentes instrucciones de uso para su correcta cumplimentación. Los cuestionarios fueron cumplimentados de forma anónima.

\section{Análisis estadístico}

Se ha realizado un análisis estadístico por medio del Statistical Package for Social Sciences 23.0 (SPSS-23.0). Se han analizado frecuencias y porcentajes, media y desviación típica de cada ítem. Además, se ha aplicado la prueba t de student para muestras independientes para comprobar la existencia o no de diferencias significativas según sexo y edad. Se utilizó $\mathrm{p}<0.05$ como nivel de significación.

\section{Resultados}

En la Tabla 1 se describen las frecuencias de respuesta de la muestra en los diferentes ítems del cuestionario agrupados por dimensiones. En cuanto a la dimensión del entrenador, un $100 \%$ de usuarios están contentos con el trato recibido por el monitor. El $95.4 \%$ cree que se presta una atención adecuada a los problemas surgidos. El 93.1\% observa unos entrenamientos adaptados a los intereses percibidos. Además, el 97.7\% considera que el monitor anima al grupo lo suficiente.

Con respecto a las instalaciones, el $86.3 \%$ cree que los vestuarios están lo suficientemente limpios. El 61.4\% considera que los vestuarios son lo suficientemente amplios, y el $86.4 \%$ ve las instalaciones limpias. En la dimensión del material deportivo, un $95.4 \%$ cree que se dispone de suficiente material para los entrenamientos. El $90.9 \%$ considera que se encuentra en buenas condiciones. Finalmente, un $72.7 \%$ de usuarios cree que el material es moderno.

Un $100 \%$ de usuarios consideran que la actividad ofertada es amena. Un $95.5 \%$ cree que las tareas desarrolladas en los entrenamientos son variadas. El 100\% de los usuarios valoran de forma positiva la puntualidad de las actividades. El $95.5 \%$ considera que a través de la actividad se logran los resultados previstos. El 95.5\% afirma que le ha resultado sencillo incorporarse a la misma.

En relación a la dimensión comunicación, el 90.9\% repara en la existencia de un buzón de sugerencias. El 79.5\% opina que la difusión de las actividades es adecuada, y un $75 \%$ considera que se actualiza permanentemente. Con respecto al personal, el $97.7 \%$ opina que el trato del personal es agradable y además hay una buena relación entre ellos (100\%).

Por último, en lo que afecta a la satisfacción, haber elegido esta piscina ha sido una buena elección para el $97.7 \%$ de los usuarios. Hay una mayoría absoluta de satisfacción con respecto a la realización de la actividad deportiva. Para finalizar, un $95.4 \%$ está satisfecho con la relación calidad-precio. 
Tabla 1. Frecuencias (y porcentajes) de respuesta por ítems y dimensiones.

\begin{tabular}{|c|c|c|c|c|c|}
\hline MONITOR/ENTRENADOR & 1 & 2 & 3 & 4 & 5 \\
\hline Estoy contento/a con el trato recibido por el monitor/entrenador. & $0(0.0)$ & $0(0.0)$ & $3(6.8)$ & $11(25)$ & $30(68.2)$ \\
\hline $\begin{array}{l}\text { Creo que presta una atención adecuada a los problemas de los usuarios desde el } \\
\text { primer día. }\end{array}$ & $0(0.0)$ & $2(4.5)$ & $4(9.1)$ & $14(31.8)$ & $24(54.5)$ \\
\hline $\begin{array}{l}\text { Creo que el monitor adapta las clases/entrenamientos a los intereses-necesidades de } \\
\text { los clientes. }\end{array}$ & $1(2.3)$ & $2(4.5)$ & $10(22.7)$ & $13(29.5)$ & 18(40.9) \\
\hline Considero que el monitor/entrenador anima suficientemente al grupo. & $0(0.0)$ & $1(2.3)$ & $11(25)$ & $10(22.7)$ & $22(50)$ \\
\hline INSTALACIONES & 1 & 2 & 3 & 4 & 5 \\
\hline Los vestuarios están suficientemente limpios. & $3(6.8)$ & $3(6.8)$ & $11(25)$ & 17(38.6) & $10(22.7)$ \\
\hline Los vestuarios son lo suficientemente amplios. & $4(9.1)$ & $13(29.5)$ & $10(22.7)$ & $12(27.3)$ & $5(11.4)$ \\
\hline Las instalaciones están suficientemente limpias. & $3(6.8)$ & $3(6.8)$ & $9(20.5)$ & $18(40.9)$ & $11(25)$ \\
\hline MATERIAL DEPORTIVO & 1 & 2 & 3 & 4 & 5 \\
\hline Se dispone de suficiente material para los entrenamientos. & $0(0.0)$ & $2(4.5)$ & $9(20.5)$ & $10(22.7)$ & $23(52.3)$ \\
\hline El material está en condiciones óptimas para su uso. & $1(2.3)$ & $3(6.8)$ & $13(29.5)$ & $11(25)$ & $16(36.4)$ \\
\hline El material es moderno. & $2(4.5)$ & $10(22.7)$ & $10(22.7)$ & $15(34.1)$ & $7(15.9)$ \\
\hline ACTIVIDADES & 1 & 2 & 3 & 4 & 5 \\
\hline La actividad es amena. & $0(0.0)$ & $0(0.0)$ & 6(13.6) & $20(45.5)$ & $18(40.9)$ \\
\hline $\begin{array}{l}\text { Las tareas que desarrolla en los entrenamientos/sesiones son lo suficientemente } \\
\text { variadas. }\end{array}$ & $0(0.0)$ & $2(4.5)$ & $3(6.8)$ & $19(43.2)$ & $20(45.5)$ \\
\hline Las actividades finalizan en el tiempo indicado. & $0(0.0)$ & $0(0.0)$ & $4(9.1)$ & $10(22.7)$ & $30(68.2)$ \\
\hline Con esta actividad obtengo los resultados que esperaba. & $0(0.0)$ & $2(4.5)$ & $7(15.9)$ & $12(27.3)$ & $23(52.3)$ \\
\hline Me ha resultado sencillo incorporarme en la actividad que participo. & $0(0.0)$ & $2(4.5)$ & $2(4.5)$ & $13(29.5)$ & $27(61.4)$ \\
\hline COMUNICACIÓN & 1 & 2 & 3 & 4 & 5 \\
\hline $\begin{array}{l}\text { Disponen las instalaciones de algún medio para transmitir sus sugerencias (buzón de } \\
\text { sugerencias, tablón de anuncios). }\end{array}$ & $1(2.3)$ & $3(6.8)$ & $12(27.3)$ & $4(9.1)$ & $24(54.5)$ \\
\hline La información sobre las actividades que se desarrollan en el centro es adecuada. & $5(11.4)$ & $4(9.1)$ & $11(25)$ & 7(15.9) & 17(38.6) \\
\hline La oferta de actividades se actualiza permanentemente & $6(13.6)$ & $5(11.4)$ & $16(36.4)$ & $6(13.6)$ & $11(25)$ \\
\hline \multicolumn{6}{|l|}{ PERSONAL DE LA ORGANIZACIÓN } \\
\hline El trato del personal de la instalación es agradable. & $0(0.0)$ & $1(2.3)$ & 6(13.6) & $10(22.7)$ & $27(61.4)$ \\
\hline Hay buena relación entre el personal de la instalación. & $0(0.0)$ & $0(0.0)$ & $9(20.5)$ & $8(18.2)$ & $27(61.4)$ \\
\hline SATISFACCIÓN GENERAL & 1 & 2 & 3 & 4 & 5 \\
\hline Haber elegido este club ha sido una buena decisión. & $0(0.0)$ & $1(2.3)$ & $4(9.1)$ & $7(15.9)$ & $32(72.7)$ \\
\hline Estoy conforme por haberme inscrito en el club & $0(0.0)$ & $1(2.3)$ & $5(11.4)$ & $10(22.7)$ & 28(63.6) \\
\hline Fue una buena decisión la de realizar actividades deportivas en este club. & $0(0.0)$ & $0(0.0)$ & $4(9.1)$ & $12(27.3)$ & 28(63.6) \\
\hline Estoy complacido por haberme inscrito en club & $0(0.0)$ & $1(2.3)$ & $5(11.4)$ & $11(25)$ & $27(61.4)$ \\
\hline Estoy satisfecho/a con la relación calidad/precio de la actividad. & $1(2.3)$ & $1(2.3)$ & $3(6.8)$ & $15(34.1)$ & $24(54.5)$ \\
\hline
\end{tabular}

$1=$ muy en desacuerdo 2 = en desacuerdo $3=$ de acuerdo $4=$ bastante de acuerdo $5=$ muy de acuerdo.

En la Tabla 2 se detallan los valores medios y desviaciones estándar de los diferentes ítems del cuestionario y dimensiones. Se percibe una satisfacción general en todas las dimensiones, pero se puede destacar una especial valoración positiva al monitor, al desarrollo de las actividades y a la relación entre el personal de la organización. 
Tabla 2. Valores medios (y desviaciones estándar) de los diferentes ítems y dimensiones.

\begin{tabular}{|c|c|}
\hline MONITOR/ENTRENADOR & Media (DE) \\
\hline Estoy contento/a con el trato recibido por el monitor/entrenador. & $4.6(0.6)$ \\
\hline Creo que presta una atención adecuada a los problemas de los usuarios desde el primer día. & $4.4(0.8)$ \\
\hline Creo que el monitor adapta las clases/entrenamientos a los intereses-necesidades de los clientes. & $4(1)$ \\
\hline Considero que el monitor/entrenador anima suficientemente al grupo. & $4.2(0.9)$ \\
\hline \multicolumn{2}{|l|}{ INSTALACIONES } \\
\hline Los vestuarios están suficientemente limpios. & $3.6(1.1)$ \\
\hline Los vestuarios son lo suficientemente amplios. & $3(1.2)$ \\
\hline Las instalaciones están suficientemente limpias. & $3.7(1.1)$ \\
\hline \multicolumn{2}{|l|}{ MATERIAL DEPORTIVO } \\
\hline Se dispone de suficiente material para los entrenamientos. & $4.2(0.9)$ \\
\hline El material está en condiciones óptimas para su uso. & $3.9(1)$ \\
\hline El material es moderno. & $3.3(1.1)$ \\
\hline \multicolumn{2}{|l|}{ ACTIVIDADES } \\
\hline La actividad es amena. & $4.3(0.7)$ \\
\hline Las tareas que desarrolla en los entrenamientos/sesiones son lo suficientemente variadas. & $4.3(0.8)$ \\
\hline Las actividades finalizan en el tiempo indicado. & $4.6(0.6)$ \\
\hline Con esta actividad obtengo los resultados que esperaba. & $4.3(0.9)$ \\
\hline Me ha resultado sencillo incorporarme en la actividad que participo. & $4.5(0.8)$ \\
\hline \multicolumn{2}{|l|}{ COMUNICACIÓN } \\
\hline Disponen las instalaciones de algún medio para transmitir sus sugerencias (buzón de sugerencias, tablón de anuncios). & $4(1.1)$ \\
\hline La información sobre las actividades que se desarrollan en el centro es adecuada. & $3.6(1.4)$ \\
\hline La oferta de actividades se actualiza permanentemente & $3.2(1.3)$ \\
\hline \multicolumn{2}{|l|}{ PERSONAL DE LA ORGANIZACIÓN } \\
\hline El trato del personal de la instalación es agradable. & $4.4(0.8)$ \\
\hline Hay buena relación entre el personal de la instalación. & $4.4(0.8)$ \\
\hline \multicolumn{2}{|l|}{ SATISFACCIÓN GENERAL } \\
\hline Haber elegido este club ha sido una buena decisión. & $4.6(0.7)$ \\
\hline Estoy conforme por haberme inscrito en el club & $4.5(0.8)$ \\
\hline Fue una buena decisión la de realizar actividades deportivas en este club. & $4.5(0.7)$ \\
\hline Estoy complacido por haberme inscrito en club & $4.4(0.8)$ \\
\hline Estoy satisfecho/a con la relación calidad/precio de la actividad. & $4.3(0.9)$ \\
\hline
\end{tabular}

$1=$ muy en desacuerdo 2 = en desacuerdo 3 = de acuerdo 4 = bastante de acuerdo 5 = muy de acuerdo.

Como se observa en las tablas 3 y 4 no hubo diferencias significativas según sexo y edad en ninguna de las dimensiones del cuestionario, lo cual indica que la satisfacción de los usuarios no varía en función del sexo o la edad. 
Tabla 3. Diferencias según sexo: Media (DE).

\begin{tabular}{|c|c|c|c|c|}
\hline Dimensión & Hombres $(\mathrm{n}=16)$ & Mujeres $(\mathrm{n}=28)$ & Dif. medias & Signific. \\
\hline Monitor/Entrenador & $4.19(0.53)$ & $4.37(0.73)$ & 0.18 & 0.400 \\
\hline Instalaciones & $3.70(0.76)$ & $3.30(1.00)$ & -0.40 & 0.200 \\
\hline Material Deportivo & $3.90(0.69)$ & $3.70(0.90)$ & -0.20 & 0.470 \\
\hline Actividades & $4.20(0.48)$ & $4.40(0.60)$ & 0.20 & 0.300 \\
\hline Comunicación & $3.90(0.80)$ & $3.50(0.90)$ & -0.40 & 0.170 \\
\hline Personal de la Organización & $4.50(0.60)$ & $4.30(0.80)$ & -0.20 & 0.460 \\
\hline Satisfacción General & $4.40(0.80)$ & $4.60(0.60)$ & 0.20 & 0.380 \\
\hline Satisfacción calidad / Precio & $4.50(0.60)$ & $4.30(1.00)$ & -0.20 & 0.450 \\
\hline TOTAL & $4.14(0.45)$ & $4.10(0.51)$ & -0.04 & 0.760 \\
\hline
\end{tabular}

${ }^{*} \mathrm{p}<0.05^{* *} \mathrm{p}<0.01$

Tabla 4. Diferencias según edad: Media (DE).

\begin{tabular}{|c|c|c|c|c|}
\hline Dimensión & $\leq 20$ años $(\mathrm{n}=23)$ & $>20$ años $(\mathrm{n}=21)$ & Dif. medias & Signific. \\
\hline Monitor/Entrenador & $4.25(0.58)$ & $4.36(0.74)$ & 0.11 & 0.597 \\
\hline Instalaciones & $3.42(1.15)$ & $3.49(0.80)$ & 0.07 & 0.814 \\
\hline Material Deportivo & $3.82(0.88)$ & $3.79(0.87)$ & -0.03 & 0.903 \\
\hline Actividades & $4.26(0.56)$ & $4.51(0.51)$ & 0.25 & 0.127 \\
\hline Comunicación & $3.80(0.92)$ & $3.48(0.93)$ & -0.32 & 0.258 \\
\hline Personal de la Organización & $4.46(0.65)$ & $4.38(0.83)$ & -0.08 & 0.739 \\
\hline Satisfacción General & $4.51(0.80)$ & $4.52(0.62)$ & 0.01 & 0.953 \\
\hline Satisfacción calidad / Precio & $4.48(0.66)$ & $4.24(1.09)$ & -0.24 & 0.378 \\
\hline TOTAL & $4.12(0.54)$ & $4.14(0.43)$ & 0.02 & 0.891 \\
\hline
\end{tabular}
${ }^{*} \mathrm{p}<0.05^{* *} \mathrm{p}<0.01$

\section{Discusión}

Kim y Trail (2010) y Bodet (2006) consideran que la calidad de los factores humanos y los factores tangibles son determinantes en la formación de la satisfacción del participante. Es por ello que, conocer el grado de satisfacción de los usuarios hacia los aspectos técnicos, la instalación, las actividades, el material y la organización y comunicación de la piscina municipal de Puente Tocinos ha sido el principal objetivo de este estudio.

El grado de satisfacción del servicio se evaluó positivamente para las dimensiones analizadas. Los valores más altos de satisfacción se dieron en las dimensiones: monitores, actividades ofertadas, relación entre el personal de la instalación, y calidad vinculada al precio. Los valores más bajos correspondieron a la limpieza y amplitud de los vestuarios de la instalación. En el estudio de Calabuig, Quintanilla y Mundana (2008), los elementos tangibles son los que obtienen peor puntuación.

Nuviala et al (2012) también obtuvieron una evaluación positiva en sus elementos analizados. De forma similar al presente estudio, los aspectos relacionados con los recursos humanos, los factores técnicos y el personal de servicio obtuvieron las puntuaciones más altas y las más bajas fueron las relacionadas con la comunicación. Nuviala et al (2012) también obtuvieron evaluaciones positivas, pero las dimensiones relacionadas con la instalación fueron las que peor valoradas estuvieron y, en cambio, la dimensión mejor valorada se obtuvo en el factor humano.

Calabuig et al, (2010) en su estudio realizado acerca del grado de satisfacción de los espectadores de atletismo, concluyeron que los espectadores mostraban una alta calidad percibida, al igual que en el presente estudio. Las dimensiones mejor valoradas por los espectadores eran la accesibilidad y los tangibles.

Vila, Sánchez y Manaserro, (2009) llevaron a cabo un estudio para analizar el grado de satisfacción de los usuarios de las instalaciones de Palma de Mallorca. Los resultados que obtuvieron fueron positivos, ya que, en general, el grado de satisfacción de los usuarios era alto, al igual que en el presente 
estudio. Los aspectos que se consideraron fueron las necesidades de los usuarios, el funcionamiento de las instalaciones, la calidad del servicio, el ajuste de los horarios y la atención de los empleados. A pesar de que el grado de satisfacción es positivo, como en el presente estudio, los resultados que obtuvieron afirmaban que los usuarios se sentían menos satisfechos con el procedimiento de gestión de quejas.

Camino y García (2014), realizaron un estudio para evaluar el valor, la calidad y la satisfacción percibida por los padres de deportistas y deportistas adultos. Los resultados mostraron como las puntuaciones más elevadas fueron relativas a la actividad y al personal, coincidiendo con los datos extraídos. Además, también en este estudio la dimensión referida a la comunicación fue de las peores valoradas.

Los resultados refuerzan la relación entre la satisfacción, la calidad percibida y el valor percibido como afirma Bernal (2014). Este dato muestra la importancia de gestionar y abordar elaboradamente todos los aspectos que puedan influir en la percepción del servicio que se analiza. Estos resultados indican que las actuaciones futuras de gestión de piscinas deben estar orientadas a potenciar aspectos como la amplitud de espacios y limpieza de los mismos. Aún así, no se debería abandonar ni dejar de lado otros importantes aspectos como las actividades que se ofrecen, los factores técnicos, la imagen de la organización y la comunicación.

Es esencial sugerir, como líneas futuras de investigación, analizar el grado de satisfacción que tienen los monitores de la instalación. Sería fundamental para obtener información que permita conocer la situación actual de cara a realizar las correcciones oportunas si fuese necesario. También destacar que, aunque la presente investigación amplía la información proporcionada previamente por investigaciones similares, hay que reconocer que la literatura acerca de esta temática sigue siendo escasa, por lo que se recomienda seguir llevando a cabo investigaciones similares en diferentes centros deportivos y en distintas áreas geográficas.

\section{Conclusión}

Los clientes externos de la piscina analizada en este estudio de caso se encuentran en general satisfechos con la instalación acuática, especialmente con el trato recibido por el monitor y su implicación con el grupo.

En relación al material, los usuarios consideran que es variado y que se encuentra en unas condiciones óptimas. Se encuentran altamente satisfechos con las actividades ofertadas, con el trato del personal de la instalación y con la elección de esta. Finalmente, todos coinciden en que la relación calidad/ precio es adecuada.

En cuanto a las variables sexo y edad, no existen diferencias significativas en la satisfacción de los usuarios. Este es un aspecto que los gestores deportivos deben tener en cuenta para mejorar la satisfacción global de los clientes con la instalación.

Se recomienda a los gestores deportivos a cargo de piscinas que presten especial atención a la amplitud de espacios como vestuarios y limpieza de los mismos, aspectos que en este estudio han mostrado ser elementos causantes de una menor satisfacción de los usuarios.

\section{Referencias bibliográficas}

1. Aparicio Sarmiento, A., Gil López, M. I., López Sánchez, G. F. \& Díaz Suárez, A. (2016). Satisfaction of users of two padel clubs in Cartagena (Region of Murcia). SPORT TK: Revista EuroAmericana de Ciencias del Deporte, 5(2), 27-32.

2. Armada, E., Martínez-Gallego, F., Segarra, E., y Díaz, A. (2016). La satisfacción del usuario como indicador de calidad en el servicio municipal de deportes. Percepción, análisis y evolución. SPORT TK: Revista Euroamericana de Ciencias del Deporte, 5(1), 119-122.

3. Bernal, A. (2014). Fidelización de clientes en organizaciones deportivas: calidad, valor percibido y satisfacción como factores determinantes. Tesis Doctoral. Universidad de Sevilla, Sevilla.

4. Bodet, G. (2006). Investigating customer satisfaction in a health club context by an application of the tetraclasse model. European Sport Management Quarterly, 6(2), 149-165.

5. Calabuig, F., Quintanilla, I., y Mundina, J. (2008). La calidad percibida de los servicios deportivos: diferencias según instalación, género, edad y tipo de usuario en servicios náuticos. Revista Internacional de Ciencias del Deporte, 10(4), 25-43.

6. Calabuig, F., Burillo, P., Crespo, J., Mundina, J., y Gallardo, L. (2010). Satisfacción, calidad y valor percibido en espectadores de atletismo satisfacción. Revista Internacional de Medicina y Ciencias de la Actividad Física y el Deporte, 10(40), 577-593.

7. Camino, M., y García, J. (2014). La percepción de calidad, valor y sa- tisfacción de un club deportivo. La perspectiva de padres y deportistas adultos. E-Balonmano.com: Revista de Ciencias del Deporte, 10(2), 99-112.

8. Castro-Sánchez, M., Zurita-Ortega, F., Chacón-Cuberos, R., Martínez-Martínez, A., Espejo-Garcés, T., \& Álvaro-González, J. I. (2015). Harmful substances and motivational climate in relation to physical activity. Health and Addictions/Saludy drogas, 15(2), 115-126.

9. Chacón Cuberos, R., Castro Sánchez, M., Caracuel Cáliz, R., Padial Ruz, R., Collado Fernández, C., \& Zurita Ortega, F. (2016). Profiles of alcohol and tobacco use among adolescents from Andalusia in the first cycle of secondary education. Health and Addictions/Saludy Drogas, 16(2), 93-104.

10. Cuadrado Cayuela, F., Gómez Arcas, E., López Sánchez, G. F. \& Díaz Suárez, A. (2015). Satisfaction of gym's users in Lorca (Region of Murcia). EFDEPORTES: Lecturas, Educación Física y Deportes, Revista Digital, 203, 1-6.

11. Espejo Garcés, T., Martínez Martínez, A., Chacón Cuberos, R., Zurita Ortega, F., Castro Sánchez, M., \& Cachón Zagalaz, J. (2017). Alcohol consumption and physical activity in adolescents from rural environment. Health and Addictions/Saludy Drogas, 17(1), 97-105.

12. García Mayor, J., Vegara Ferri, J. M., López Sánchez, G. F., Díaz Suárez, A. (2016). Satisfaction of sports services users in Orihuela (Alicante). SPORT TK: Revista EuroAmericana de Ciencias del Deporte, 5(Supl.), 155-162. 
13. Kim, M., Trail, G. (2010). The effects of service provider employment status and ervicequality exchange on perceived organizational image and purchase intention. Sport Management Review, 13(3), 225234.

14. López Sánchez, G. F. (2016). Management and Economics of Sport. SPORT TK-Revista Euroamericana de Ciencias del Deporte, 5(1), 101101.

15. López Sánchez, G. F., González Víllora, S. \& Díaz Suárez, A. (2016a). Level of habitual physical activity in children and adolescents from the Region of Murcia (Spain). SpringerPlus, 5:386, 1-6. http://dx.doi. org/10.1186/s40064-016-2033-8

16. López Sánchez, G. F., Ahmed, D., Borrego Balsalobre, F. J., López Sánchez, L. \& Díaz Suárez, A. (2016b). Level of habitual physical activity in 8-9 years old schoolchildren from Spain and India. MHSalud: Revista en Ciencias del Movimiento Humano y Salud, 12(2), 1-10. http:// dx.doi.org/10.15359/mhs.12-2.3

17. López Sánchez, G. F., Ahmed, D. \& Díaz Suárez, A. (2017). Level of habitual physical activity among 13-year-old adolescents from Spain and India. A cross-cultural study. SPORT TK: Revista EuroAmericana de Ciencias del Deporte, 6(1), 67-74.

18. López Sánchez, G. F. (2017). Composición corporal, imagen corporal, actividad física y salud en niños y adolescentes. Tesis Doctoral. Universidad de Murcia, Espańa.

19. López-Sánchez, G. F., Díaz-Suárez, A., Radzimiński, Ł., \& Jastrzębski, Z. (2017). Effects of a 12-week-long program of vigorous-intensity physical activity on the body composition of 10-and 11-year-old children. Journal of Human Sport and Exercise, 12(1), 235-244. doi:10.14198/ jhse.2017.121.19

20. Martínez-Moreno, A. (2010). Diagnóstico de excelencia en la gestión de los servicios deportivos municipales: modelo EFQM. Tesis Doctoral. Universidad de Murcia, Espańa.

21. Nuviala A.; Tamayo J., y Nuviala, R. (2012). Perceived quality of the school sport as predictor of sports dropout in teenagers. Revista Internacional de Medicina y Ciencias de la Actividad Física y el Deporte. 12(47). 389-404.
22. Nuviala, A., Grao-Cruces, A., Tamayo, J.A., Nuviala, R., Álvarez, J., y Fernández-Martínez, A. (2013). Diseño y análisis del cuestionario de valoración de servicios deportivos (EPOD2). Revista Internacional de Medicina y Ciencias de la Actividad Física y el Deporte, 13 (51), 419-436.

23. Rial, A., Alonso, D., Rial, J., Picón, E., \& Varela, J. (2009). Un intento de segmentación integral de los usuarios de centros deportivos. Apunts: Educación Física y Deportes, (95), 82-91.

24. Reynoso Vergara, C., Riquelme López, Y. P., López Sánchez, G. F., Díaz Suárez, A. (2016). Job Satisfaction of Sports Instructors in Pilar de la Horadada (Alicante). EFDEPORTES: Lecturas, Educación Física y Deportes, Revista Digital, 213, 1-9.

25. Rodríguez Carrasco, F. J., Ros Saura, J. C., López Sánchez, G. F. \& Díaz Suárez, A. (2015). Satisfacción de usuarios de gimnasio en los Alcázares (Región de Murcia). Sport Managers: Revista profesional de la salud e instalaciones deportivas, 98, 36-40.

26. Rodríguez, G., y Barriopedro, M.I. (2003). Niveles de satisfacción en usuarios de piscinas cubiertas con la profundidad del vaso utilizado para la práctica. Revista de Psicología del Deporte, 12(2), 147-164.

27. Smith, L., Fisher, A., \& Hamer, M. (2015). Television viewing time and risk of incident obesity and central obesity: the English longitudinal study of ageing. BMC Obesity, 2(1), 12. doi: 10.1186/s40608-0150042-8

28. Vespalec, T., Pavlík, J., Zvonař, M. \& Zeman, T. (2016). Physical activity of Czech schoolchildren in the autumn season. Journal of Human Sport and Exercise, 11(Proc1), S137-S145. doi:10.14198/jhse.2016.11. Proc1.04

29. Vila, I., Sánchez, C., y Manassero, A. (2009). Satisfacción percibida de los usuarios de las instalaciones deportivas municipales de Palma de Mallorca. Revista Iberoamericana de Psicología del Ejercicio y el Deporte, 4(1), 59-73.

30. Williams, G., Aggio, D., Stubbs, B., Pardhan, S., Gardner, B., \& Smith L. (2017). Physical activity levels in children with sensory problems: Cross-sectional analyses from the Millennium Cohort Study. Disability and Health Journal, In Press. doi: 10.1016/j.dhjo.2017.07.002 